\title{
Roseburia intestinalis supernatant ameliorates colitis induced in mice by regulating the immune response
}

\author{
WEIWEI LUO ${ }^{1,2}$, ZHAOHUA SHEN $^{1,2}$, MINZI DENG $^{1}$, XIAYU LI $^{1,2}$, BEI TAN $^{1,2}$, MENGWEI XIAO $^{1,2}$, \\ SHUAI WU ${ }^{1,2}$, ZHENYU YANG ${ }^{1,2}$, CHANGXIN ZHU ${ }^{1,2}$, LI TIAN $^{1}$, XING WU $^{1,2}$, \\ XIANGRUI MENG ${ }^{1,2}$, YONGSHENG QUAN ${ }^{1,2}$ and XIAOYAN WANG ${ }^{1,2}$ \\ ${ }^{1}$ Department of Gastroenterology, The Third Xiangya Hospital, Central South University, Changsha, Hunan 410013; \\ ${ }^{2}$ Hunan Key Laboratory of Non-Resolving Inflammation and Cancer, Changsha, Hunan 410008, P.R. China
}

Received November 20, 2018; Accepted April 15, 2019

DOI: $10.3892 / \mathrm{mmr} .2019 .10327$

\begin{abstract}
Inflammatory bowel disease (IBD), which includes ulcerative colitis (UC) and Crohn's disease (CD), has a complex etiology that may be associated with dysbiosis of the microbiota. Previously, our study revealed significant loss of Roseburia intestinalis from the gut of untreated patients with $\mathrm{CD}$, and that $R$. intestinalis exerted anti-inflammatory functions in TNBS-induced colitis; however, the function of R.intestinalis supernatant is unknown. Therefore,LPS-induced macrophages, including RAW264.7 macrophages and bone marrow-derived macrophages were treated with $R$. intestinalis supernatant. The results indicated that $R$. intestinalis supernatant suppressed expression of interleukin (IL)-6 and signal transducer and activator of transcription 3 (STAT3) by macrophages. Additionally, these findings were further verified in vivo in DSS- and TNBS-induced mouse models of colitis. It was observed that $R$. intestinalis supernatant ameliorated IBD colitis by reducing the number of inflammatory macrophages and Th17 cells in the colon, and by downregulating the expression of IL-6 and STAT3. Finally, the non-protein components of $R$. intestinalis supernatant were examined using gas chromatography-mass spectrometry analysis and identified the presence of short-chain fatty acids. In conclusion, the results of the present study indicated that $R$. intestinalis supernatant may regulate immune responses and ameliorate colitis.
\end{abstract}

\section{Introduction}

Inflammatory bowel disease (IBD), which includes Crohn's disease (CD) and ulcerative colitis (UC), is a chronic and non-resolving intestinal inflammatory disease with poor

Correspondence to: Professor Xiaoyan Wang, Department of Gastroenterology, The Third Xiangya Hospital, Central South University, 138 Tongzipo Road, Changsha, Hunan 410013, P.R. China E-mail: wangxiaoyan2913@126.com

Key words: Roseburia intestinalis, supernatant, inflammatory bowel disease, macrophage, Th17 cells, short-chain fatty acids prognosis $(1,2)$. A recent study has shown that genetic and environmental factors, and dysbiosis of the microbiota serve important roles in the pathogenesis of IBD (3). The intestinal microbiota was determined to be closely associated with IBD. Our previous studies revealed that the intestinal population of Roseburia intestinalis (R. intestinalis) in untreated patients with CD was markedly lower than in healthy controls $(4,5)$. $R$. intestinalis is a butyrate-producing Gram-positive bacteria belonging to Clostridium subphylum cluster XIVa (6). Previously, our study demonstrated that $R$.intestinalis increased the abundance of T regulatory (Treg) cells and upregulated the expression of cytokines thymic stromal lymphopoietin (TSLP) and transforming growth factor- $\beta$ (TGF- $\beta$ ) to ameliorate intestinal inflammation (4); however, whether $R$. intestinalis supernatant has a beneficial effect on IBD requires further investigation.

The microbiota has a direct effect on host immunity; metabolites secreted by the gut microbiota serve a role in innate immune responses (7). Important metabolites include indoles, folate, trimethylamine-N-oxide, and short-chain fatty acids (SCFAs); among these, SCFAs have been the subject of much research (7). Gut microbes ferment dietary fiber to generate SCFAs, specifically butyrate, which regulates the development and activity of innate and adaptive immune cells that are crucial for protecting the host from intestinal injury (8). Dysbiosis of the microbiota and reduced concentrations of SCFAs are associated with a significant increase in the number of pro-inflammatory immune cells in the intestinal mucosa of patients with IBD (9). Butyrate promotes the secretion of anti-inflammatory cytokines by colonic macrophages and dendritic cells by binding to hydroxycarboxylic acid receptor 2, thereby inducing the differentiation of Treg cells and interleukin (IL)-10-producing T cells to ameliorate 2,4,6-trintirobenzenesulfonic acid (TNBS)-induced intestinal inflammation (10). A recent study demonstrated that Faecalibacterium prausnitzii, a commensal bacterium abundant in the human gut, produces butyrate to maintain the Th17/Treg balance and suppress colitis (11).

In the present study, the effects of $R$. intestinalis supernatant on LPS-induced macrophages were investigated in vitro, and its role in dextran sulfate sodium (DSS)- and TNBS-induced colitis was determined in vivo. In addition, the 
non-protein components of the supernatant were analyzed via gas chromatography-mass spectrometry (GC-MS) analysis. To the best of our knowledge, this is the first study to examine the effects of $R$. intestinalis supernatant on colitis. The results may provide insight into the mechanism by which the commensal microbiota and its metabolites interact with the host, and may facilitate the development of novel therapeutic strategies for treating IBD.

\section{Materials and methods}

Animals. Male C57BL/6 mice (6-weeks-old, 17-18 g) were obtained from the laboratory animals department of Central South University (Changsha, China) and housed in specific pathogen-free conditions at $22-26^{\circ} \mathrm{C}$, under a $12: 12-\mathrm{h}$ light/dark cycle with $40-70 \%$ humidity and ad libitum access to food and water. All animal experiments were approved by the Ethics Committee of Medical Research of Central South University and were conducted in accordance with the National Institutes of Health Guide for the Care and Use of Laboratory Animals (12).

Bacterial culture. $R$. intestinalis DSMZ-14610 was grown as previously described (4). $R$. intestinalis supernatant was collected after centrifugation at $2,000 \mathrm{x} \mathrm{g}$ and $4^{\circ} \mathrm{C}$ for $20 \mathrm{~min}$ and passed through a $0.22-\mu \mathrm{m}$ sterile filter (EMD Millipore) to remove bacteria. Sterile culture medium was used as placebo. R. intestinalis supernatant and fresh sterile BD BACTEC $^{\text {Tм }}$ Lytic/10 Anaerobic/F medium (BD Diagnostics; Becton-Dickinson and Company) were frozen at $-80^{\circ} \mathrm{C}$ until required. Medium and supernatant were lyophilized and diluted with PBS by a factor of two or five prior to administration.

Induction of colitis with DSS. Mice $(\mathrm{n}=24)$ were randomly divided into three groups ( $\mathrm{n}=8$ per group): The control, colitis (DSS), and DSS and R. intestinalis supernatant (DSS + SUP) groups. Between days 0-7, all mice (except the control group) received 3\% DSS (MW, 36,000-50,000; MP Biomedicals). In addition, the control and colitis groups received $0.2 \mathrm{ml}$ of 5X R. intestinalis culture medium (BD Diagnostics; Becton-Dickinson and Company) by gavage, and the DSS + SUP group was treated with $0.2 \mathrm{ml}$ of $5 \mathrm{X} R$. intestinalis supernatant by gavage once per day following DSS treatment from day 0 (for 7 days in total). Mice were euthanized for tissue collection on day 7 .

Induction of colitis with TNBS. Mice were assigned to similar groups as aforementioned (control, TNBS and TNBS + SUP). For the induction of colitis, mice were pre-sensitized by applying $150 \mu \mathrm{l}$ of $5 \%$ TNBS (Sigma-Aldrich; Merck KGaA) in a $4: 1 \mathrm{v} / \mathrm{v}$ ratio with acetone/olive oil (ratio of 4:1 v/v) mixture onto the shaved dorsal skin mice for 1 day. Control mice were treated with acetone/olive oil mixture alone (day 1) (4). On days 8 and 10, mice were anesthetized with isoflurane and administered $100 \mu 1$ TNBS solution [5\% TNBS in 50\% ethanol (1:1 v/v ratio)] via the rectum. Mice were held upside down for 1 min after administration of TNBS. The control group received $50 \%$ ethanol instead of TNBS. The control and colitis groups received $0.2 \mathrm{ml}$ of $5 \mathrm{X} R$. intestinalis culture medium
(BD Diagnostics; Becton-Dickinson and Company), and the TNBS + SUP group received $R$. intestinalis supernatant via gavage once a day from day 10 (for 5 days in total). Mice were euthanized for tissue collection on day 14 following pre-sensitization.

Disease activity index. The severity of colitis was assessed by measuring body weight loss, stool characteristics and the occurrence of hematochezia using the three-component mouse disease activity index (DAI; $n=8$; Table SI), as described previously (5).

Cell isolation and flow cytometry. Murine bone marrow macrophages (BMMs) were cultured as described (13), with certain modified as thus described: Briefly, the mouse femur and tibia were separated and both ends were cut. Then, the bone marrow was flushed with 1X PBS using a 1-ml syringe. Cells $\left(\sim 2 \times 10^{6} / \mathrm{ml}\right)$ were cultured in 6 -well plates in complete RPMI-1640 containing $10 \mathrm{ng} / \mathrm{ml}$ recombinant macrophage colony stimulating factor (PeproTech, Inc.). Cells were used on day 5 , at which point $\sim 70 \%$ were assessed for $\mathrm{F} 4 / 80^{+} \mathrm{CD} 11 b^{+}$by fluorescence activated cell sorting (FACS) analysis. To prepare peritoneal macrophages, mice were sacrificed and $5 \mathrm{ml}$ of RPMI-1640 was injected into the peritoneal cavity. The fluid was collected and centrifuged at $240 \mathrm{x} g$ and room temperature for $5 \mathrm{~min}$. To isolate lamina propria cells, colon tissue was treated with a pre-digestion solution [1X Hank's Balanced Salt Solution $+5 \mathrm{mM}$ EDTA $+1 \mathrm{mM}$ dithiotreithol $+5 \%$ fetal bovine serum (Biological Industries)] at $37^{\circ} \mathrm{C}$ for $20 \mathrm{~min}$ to remove epithelial cells. Then, the tissue was treated with digestion solution [0.3 $\mathrm{mg} / \mathrm{ml}$ type IV collagenase (Sigma-Aldrich; Merck KGaA), 3 mg/ml Dispase II (Roche Diagnostics GmbH) and $0.25 \mathrm{mg} / \mathrm{ml}$ DNase I (Sigma-Aldrich; Merck KGaA)] at $37^{\circ} \mathrm{C}$ for $20 \mathrm{~min}$ with gentle agitation. Cells were collected and purified via a Percoll gradient $(40 / 80 \%)$ prior to FACS analysis. Subsequently, cells were incubated with anti-mouse antibodies specific for F4/80 (1:50; cat. no. 123113; phycoerythrin-cyanine 7 conjugate; BioLegend, Inc.) and CD11b (1:50; cat. no. 101205; FITC conjugate; BioLegend, Inc.) at $4{ }^{\circ} \mathrm{C}$ for 30 min to detect macrophage, or with Leukocyte Activation Cocktail, BD GolgiPlug ${ }^{\mathrm{TM}}$ (BD Biosciences) at $37^{\circ} \mathrm{C}$ for $5 \mathrm{~h}$ to elicit a primary cytokine response from $\mathrm{T}$ cells. The $\mathrm{T}$ cells were stained with anti-mouse Abs specific for IL-17 (1:50; cat. no. 146307; allophycocyanin conjugate; BioLegend, Inc.) and CD4 markers (1:100; cat. no. 130308; FITC conjugate; BioLegend, Inc.) at $4^{\circ} \mathrm{C}$ for $30 \mathrm{~min}$ prior to Th17 analysis. Flow cytometry was performed using a FACS Arial II flow cytometer (BD Biosciences) and analyzed using FlowJo 7.0 FACS software (FlowJo LLC).

Histology and immunohistochemistry (IHC). For H\&E detection, proximal and distal colon tissues were fixed in $4 \%$ phosphate-buffered formaldehyde solution at $4{ }^{\circ} \mathrm{C}$ for $24 \mathrm{~h}$, embedded in paraffin, cut into $5 \mu \mathrm{m}$-thick sections, and stained with hematoxylin for $30 \mathrm{sec}$ and eosin for $2 \mathrm{~min}$ (both at room temperature). The slides were scored for inflammation by two pathologists (blinded to the treatment regimens) using previously described criteria (14) (Table SII). For IHC $(n=6)$, $5 \mu \mathrm{m}$-thick formalin-fixed paraffin-embedded specimens of the distal colon were boiled in sodium citrate solution $(0.01 \mathrm{M}$, 
pH 6.0; Wuhan Goodbio Technology Co., Ltd.) at $100^{\circ} \mathrm{C}$ for $20 \mathrm{~min}$ and then cooled to room temperature. Sections were incubated at $4^{\circ} \mathrm{C}$ overnight with an anti-phosphorylated (p)-STAT3 rabbit mAb (1:200; cat. no. 9145; Cell Signaling Technology, Inc.) and an anti-IL-6 rabbit polyclonal Ab (1:200; cat. no. 21865-1-AP; ProteinTech Group, Inc.), followed by incubation with corresponding secondary biotin-conjugated Abs (cat. no. KIT-9710, Mai New Biotechnology Development Company) at $37^{\circ} \mathrm{C}$ for $1 \mathrm{~h}$ according to the manufacturer's protocols. The procedures were performed using a 3',3'-diaminobenzidene kit (Mai New Biotechnology Development Company) and scored using a light microscope (magnification, x100; DP72; Olympus Corporation) by two independent pathologists in a blinded manner, with at least 5 high powered fields counted per sample.

Reverse transcription-quantitative polymerase chain reaction ( $q P C R)$. Total RNA from colon tissue or cells was isolated by using TRIzol ${ }^{\circledR}$ (Thermo Fisher Scientific, Inc.), and cDNA was synthesized using a RevertAid First Strand cDNA Synthesis kit (Thermo Fisher Scientific, Inc.) according to the manufacturer's protocols. cDNA was amplified by qPCR using SYBR ${ }^{\circledR}$-Green Supermix (Vazyme) and a Bio-Rad CFX96 Real-Time PCR Detection System (Bio-Rad Laboratories, Inc.). The thermocycling conditions for qPCR were: $95^{\circ} \mathrm{C}$ for $30 \mathrm{sec}$, followed by 40 cycles at $95^{\circ} \mathrm{C}$ for $5 \mathrm{sec}$ and $60^{\circ} \mathrm{C}$ for $30 \mathrm{sec}$. The relative expression of target gene mRNA was calculated using the $2^{-\Delta \Delta \mathrm{Cq}}$ method (15) and GAPDH was used as the reference gene (primer sequences are presented in Table SIII).

Western blotting. Total protein was extracted from colon tissues with lysis buffer and centrifuged at $13,000 \mathrm{x}$ g and $4^{\circ} \mathrm{C}$ for $10 \mathrm{~min}$. Protein concentration was determined by a $\mathrm{BCA}$ assay (Thermo Fisher Scientific, Inc.). Protein samples $(30 \mu \mathrm{g}$; $1 \mu \mathrm{g} / \mu \mathrm{l})$ were boiled for $5 \mathrm{~min}$ at $100^{\circ} \mathrm{C}$, separated via $10 \%$ SDS-PAGE, and transferred onto polyvinylidene difluoride membranes (Merck KGaA). Membranes were blocked with $5 \%$ bovine serum albumin (Wuhan Goodbio Technology Co., Ltd.) at room temperature for $1 \mathrm{~h}$, and then incubated with primary Abs, including GAPDH mouse mAb (1:2,000; cat. no. 60004-1-Ig; ProteinTech Group, Inc.), p-STAT3 rabbit mAb (1:1,000; cat. no. 9145; Cell Signaling Technology, Inc.) and STAT3 mouse mAb (1:1,000; cat. no. 9139; Cell Signaling Technology, Inc.) at $4^{\circ} \mathrm{C}$ overnight. Subsequently, membranes were incubated with corresponding secondary horseradish peroxidase (HRP)-conjugated anti-mouse Abs (1:5,000; cat. no. SA00001-1; ProteinTech Group, Inc.) or HRP-conjugated anti-rabbit Abs (1:5,000; cat. no. SA00001-2; ProteinTech Group, Inc.) for $1 \mathrm{~h}$ at $37^{\circ} \mathrm{C}$, and then developed with an enhanced chemiluminescence detection system (Bio-Rad Laboratories, Inc.).

ELISA. Peripheral blood was collected from mice and centrifuged at $2,000 \mathrm{x} \mathrm{g}$ at room temperature for $15 \mathrm{~min}$ to obtain mouse serum for the following experiments. The concentration of IL-6 and tumor necrosis factor- $\alpha$ (TNF- $\alpha$ ) in mouse serum and the cell-culture supernatant was quantified using ELISA kits (cat. nos. 88-7064-22 and 88-7324-22; eBioscience; Thermo Fisher Scientific, Inc.) according to the manufacturer's recommendations.
GC-MS analysis of SCFAs. The mouse cecal samples was prepared as described by Turnbaugh et al (16), and the supernatant samples was prepared as described by Zhou et al (11). GC-MS analysis of concentrated bacterial culture supernatant and mouse cecal samples was performed using an Agilent 6890N/5975B GC-MS instrument (Agilent Technologies, Inc.) fitted with an Agilent HP-INNOWAX column (30 m x0.25 mm; internal diameter, $0.25 \mu \mathrm{m})$. All samples were collected, frozen and stored under vacuum at $-80^{\circ} \mathrm{C}$ until use. Samples were assayed using the method of Samuel and Gordon (17). Prepared samples $(1 \mu \mathrm{l})$ were used for detection, $50 \mu \mathrm{g} / \mathrm{ml}$ isocaproic acid was used as an internal standard and isocaproic acid/diethyl ether (ratio of 1:4 v/v) mixture was used as the solvent. The flow rate was $1 \mathrm{ml} / \mathrm{min}$.

In vitro inflammation model using BMCs and RAW264.7 cells. The human colon epithelial cell line Caco2 and the mouse macrophage cell line RAW264.7 were acquired from the Cancer Research Institute of Central South University. Cells were cultured at $37^{\circ} \mathrm{C}$ in $5 \% \mathrm{CO}_{2}$. in RPMI-1640 (HyClone; GE Healthcare Life Sciences) supplemented with $10 \%$ fetal bovine serum (Biological Industries). For the in vitro inflammation model, Caco2 cells, BMCs and RAW264.7 macrophages were induced by exposure to $1 \mu \mathrm{g} / \mathrm{ml}$ lipopolysaccharide (LPS; Sigma-Aldrich; Merck KGA), LPS + medium, LPS $+R$. intestinalis supernatant or LPS + sodium butyrate at $37^{\circ} \mathrm{C}$ for 12 or $24 \mathrm{~h}$; PBS was used as the control treatment. Then, macrophages were co-treated with $R$. intestinalis supernatant $(2 \mathrm{X})$ at $37^{\circ} \mathrm{C}$ for 12 or $24 \mathrm{~h}$.

Statistical analysis. Data were expressed as the mean \pm standard deviation of at least three independent experiments. $\mathrm{P}<0.05$ was considered to indicate a statistically significant difference. Statistical significance was analyzed by one-way ANOVA with a Student-Newman-Keuls post-hoc test (SPSS 18.0; SPSS, Inc.).

\section{Results}

$R$. intestinalis supernatant suppresses proinflammatory cytokine expression via LPS-induced macrophages in vitro. It has been shown that $R$. intestinalis bacteria have significant anti-inflammatory effects in the context of TNBS-induced colitis (4); however, analysis tends to be conducted using direct culture systems or with bacteria that were in contact with intestinal epithelial cells (IECs). To the best of our knowledge, no studies have investigated the effects of components secreted by $R$. intestinalis on the culture supernatant. Therefore, in vitro analyses were conducted to examine the possible anti-inflammatory effects of $R$. intestinalis supernatant on LPS-induced macrophages and $\mathrm{Caco} 2$ colonic cells. First, the effects of $R$. intestinalis supernatant (2X raw supernatant) on LPS-induced RAW264.7 macrophages were determined. The results revealed that the mRNA expression of IL-6, STAT3 and TNF- $\alpha$ by RAW264.7 cells were markedly increased upon exposure to LPS compared with blank treatment (Fig. 1A-C); however, the expression of IL-6 and STAT3, but not TNF- $\alpha$, significantly decreased in cells treated with LPS $+R$. intestinalis supernatant compared with LPS alone and LPS + medium (Fig. 1A-C). ELISA of IL- 6 and TNF- $\alpha$ concentrations yielded similar results (Fig. 1D and E). The effects of 

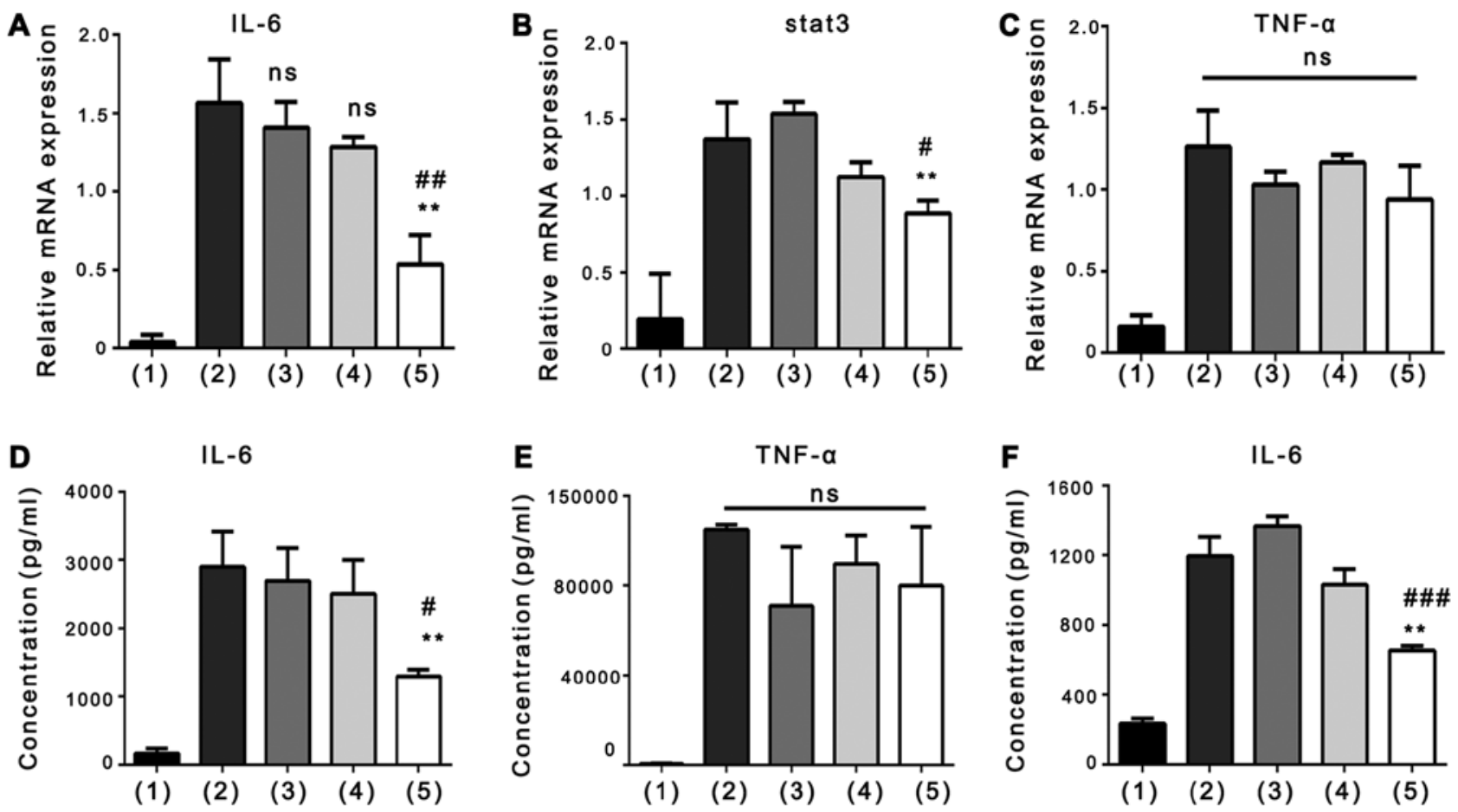

(1) Blank (2) LPS

(3) LPS+PBS

(4) LPS $+M$ (5) LPS+S2

Figure 1. Effects of $R$. intestinalis supernatant on macrophages in vitro. Reverse transcription-quantitative polymerase chain reaction analysis of (A) IL-6, (B) STAT3 and (C) TNF- $\alpha$ in RAW264.7 cells induced for $24 \mathrm{~h}$ with LPS and then treated with PBS, M or S2. (D and E) ELISA of IL-6 and TNF- $\alpha$ levels in the supernatant of RAW264.7 cells exposed to LPS for $24 \mathrm{~h}$. (F) ELISA of IL-6 in the supernatant of bone marrow macrophage cells (isolated from normal C57BL $/ 6$ mice) exposed to LPS for $12 \mathrm{~h}$. Data are representative of three independent experiments. Error bars represent mean \pm standard error of the mean. ${ }^{* *} \mathrm{P}<0.01$ vs. LPS; ${ }^{~} \mathrm{P}<0.05,{ }^{\# \#} \mathrm{P}<0.01$ and ${ }^{\# \#} \mathrm{P}<0.001$ vs. LPS + M; ns, non-significant; IL, interleukin; LPS, lipopolysaccharide; M, medium; S2, 2X R. intestinalis supernatant; STAT3, signal transducer and activator 3; TNF- $\alpha$, tumor necrosis factor- $\alpha$.

$R$. intestinalis supernatant on LPS-induced Caco2 colon cells were also examined; no significant alterations in IL-6 concentration were observed (Fig. S1A). Additionally, similarly with the results obtained for RAW264.7 cells, $R$. intestinalis supernatant led to a significant reduction in the protein expression of IL- 6 by LPS-treated BMMs compared with the LPS and LPS + medium groups (Fig. 1F). These results indicated that $R$. intestinalis supernatant inhibited the inflammatory response via LPS-induced macrophages in vitro.

$R$. intestinalis supernatant suppresses colitis in murine models of DSS- and TNBS-induced IBD. The effects of $R$. intestinalis supernatant on mouse models of DSS- and TNBS-induced colitis were investigated (Fig. 2A and B), both of which are well-established experimental models of colitis used to observe the therapeutic effects of drugs (18).

From day 5 post-treatment with supernatant, the DAI score of the DSS + SUP group of mice was significantly lower, and the colon length was longer than that of the DSS group (Fig. 2C-E). In addition, the DAI score of mice in the TNBS + SUP group improved significantly faster, and the colon length was notably longer than that of the TNBS group (Fig. 2F-H).

Then, the severity of colonic inflammation and ulcers was analyzed by H\&E staining of tissue sections. The results revealed that the distal or mid colon of non-colitic controls exhibited normal crypts and no infiltration by polymorphonuclear cells; however, polymorphonuclear cell infiltration, crypt damage, mucosal injury, edema and other inflammatory features were observed in mice treated with DSS or
TNBS (Fig. 3A and B). Intestinal inflammation was clear throughout the mid and distal colon; however, treatment with $R$. intestinalis supernatant alleviated these clinical signs, resulting in a significant reduction in the histological inflammation score compared with the DSS and TNBS groups (Fig. 3C and D). Thus, $R$. intestinalis supernatant was suggested to reduce the DAI score, prevent colon shortening and alleviate colonic inflammation in both IBD models.

R. intestinalis supernatant alters the activity of inflammatory macrophages and the production of proinflammatory cytokines. To explore alterations in immune cell activity, the abundance of $\mathrm{F} 4 / 80^{+} \mathrm{CD} 11 \mathrm{~b}^{+}$macrophages, which are recruited to sites of inflammation and are regarded as activated inflammatory macrophages, were investigated; these cells produce nitric oxide (NO) and other inflammatory mediators, such as IL-6 and TNF- $\alpha(19,20)$. Such macrophages contribute, at least in part, to the pathogenesis of IBD $(21,22)$. A significant increase in the abundance of $\mathrm{F} 4 / 80^{+} \mathrm{CD} 11 \mathrm{~b}^{+}$macrophages was detected in the DSS and TNBS groups compared with the control. A significant reduction was observed in the number of colonic macrophages in the TNBS + SUP group compared with the model, but not in the DSS + SUP group (Fig. 4A and C). In addition, the number of peritoneal macrophages in DSS and TNBS mice treated with $R$. intestinalis supernatant significantly decreased compared with the respective models (Fig. 4B and D).

DSS and TNBS treatment increased the serum concentrations of IL- 6 and TNF- $\alpha$ in both models; however, a significant 
A

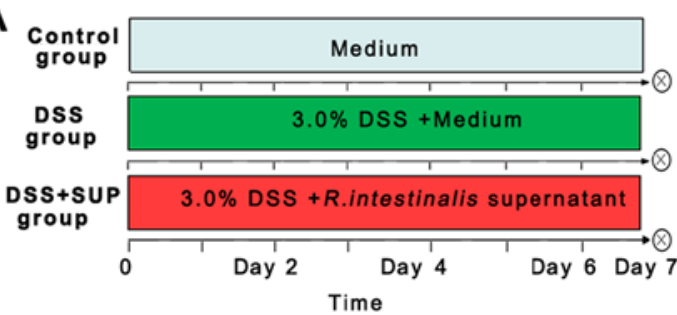

C

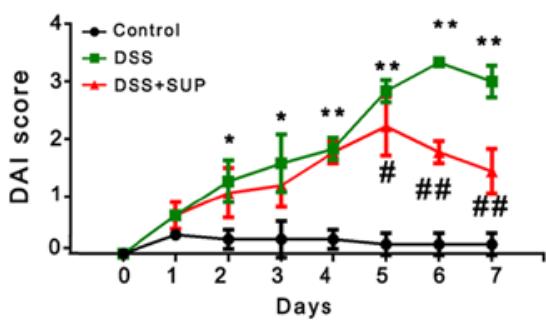

F

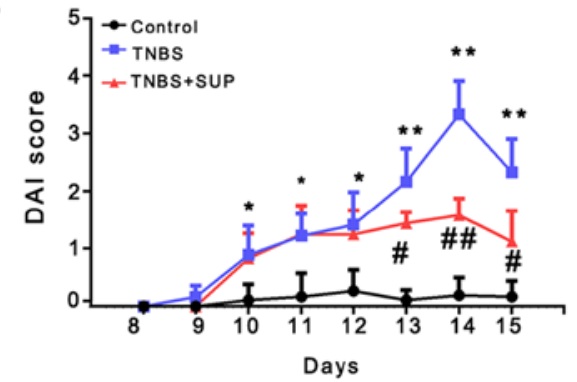

B

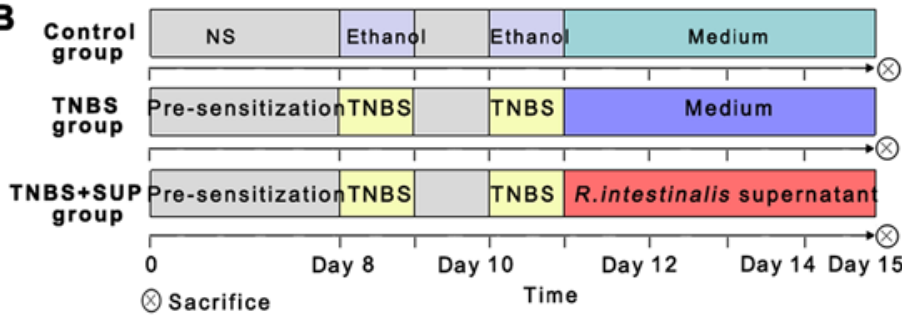

D

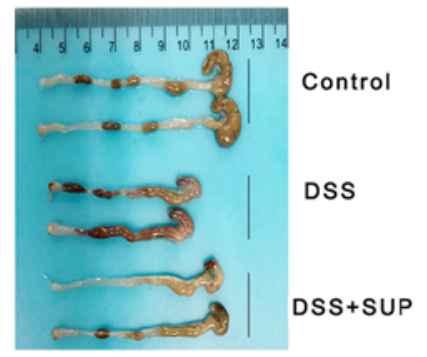

E

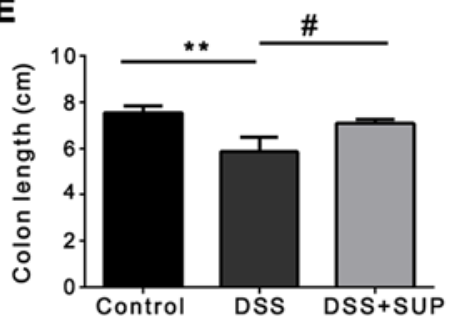

G

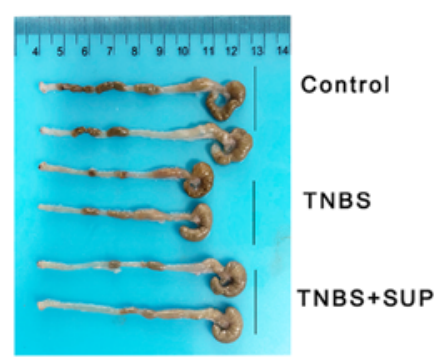

H

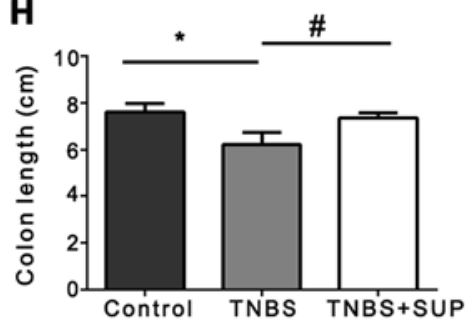

Figure 2. $R$. intestinalis supernatant improves the clinical signs and reduces colon shortening in mice with DSS- and TNBS-induced colitis. Flow diagram showing the generation of the (A) DSS-induced and (B) TNBS-induced colitis model in mice, and treatment with $R$. intestinalis supernatant or control medium. (C) DAI score of control, DSS, and DSS + SUP mice $(n=8)$. (D and E) Representative images of colons and histograms of the colon length for each group (F) DAI score of control, TNBS and TNBS + SUP mice $(n=8)$. ( $\mathrm{G}$ and $\mathrm{H})$ Representative images of colons, and histograms of the colon length for the different groups. Data are representative of three independent experiments. Error bars represent mean \pm standard error of the mean. ${ }^{*} \mathrm{P}<0.05,{ }^{* *} \mathrm{P}<0.01$ vs. control. ${ }^{\#} \mathrm{P}<0.05,{ }^{\# \#} \mathrm{P}<0.01$ vs. DSS or TNBS; ns, non-significant; DAI, disease activity index; DSS, dextran sulfate sodium; TNBS, 2,4,6-trintirobenzenesulfonic acid; SUP, supernatant treatment.

decrease in the serum levels of IL-6 was observed following treatment with $R$. intestinalis supernatant (Fig. 4E and F). Previous studies have suggested that IL-6 and STAT3 are potential therapeutic targets in IBD (23). In line with reductions in IL-6 expression in the colon, downregulation of STAT3 was also observed. The results of IHC revealed that expression of IL-6 and p-STAT3 (the active form of STAT3) in the DSS and TNBS groups markedly increased, whereas that in the DSS/TNBS + SUP groups decreased (Fig. 4G and H). Western blotting also demonstrated that the expression of STAT3 and p-STAT3 notably decreased in the DSS/TNBS + SUP groups compared with the respective model groups (Fig. 4I). Collectively, these results indicated that $R$. intestinalis supernatant inhibited inflammatory macrophages and the IL-6/STAT3 signaling pathway.

$R$. intestinalis supernatant inhibits Th17 cell differentiation. In addition to inflammatory macrophages, infiltration of the colon by $\mathrm{CD}^{+} \mathrm{T}$ cells contributes to the pathogenesis of IBD (24). CD and ulcerative colitis (UC) are associated with distinct populations of Th cells: High Th1 cell numbers are observed in the injured intestinal lamina propria of patients with CD, whereas Th2 cells are observed in UC (24). In addition, Th17 cells have been associated with CD and UC (25). A significant increase in Th17 cell numbers in the DSS and TNBS groups was reported compared with the control; however, a significant reduction was observed in model groups treated with $R$.intestinalis (Fig. 5A and B). On the contrary, no significant changes in the overall numbers of Th1 and Th 2 cells were observed between the model and $R$. intestinalis-treated groups (Fig. 5C and D). IL-6 is a pleiotropic cytokine that functions as a central regulator of the adaptive immune response in IBD; it can also serve as a promoter of Th17 cell differentiation $(11,26)$. Inhibition of IL-6 expression may be the cause of depleted Th17 cell numbers. Thus, $R$. intestinalis supernatant was proposed to inhibit the differentiation of $\mathrm{CD}^{+} \mathrm{T}$ cells, particularly Th17 cells.

$R$. intestinalis produces SCFAs, including butyrate. Finally, GC-MS analysis was conducted to examine whether non-protein components in the bacterial supernatant serve a role in regulating anti-inflammatory responses. Propionic acid, isobutyric acid, butyric acid, isovaleric acid, valeric acid and hexanoic acid were detected, and the presence of SCFAs in the R. intestinalis supernatant was confirmed (data not shown). In addition, SCFAs in the cecal contents of mice were determined. A notable increase in the levels of SCFAs in the DSS/TNBS + SUP groups was observed compared with in the DSS and TNBS groups, although the differences were not statistically significant (Fig. 6A and B). The results also revealed that propionic acid and butyric acid were the most frequently detected SCFAs in the samples, which may be 

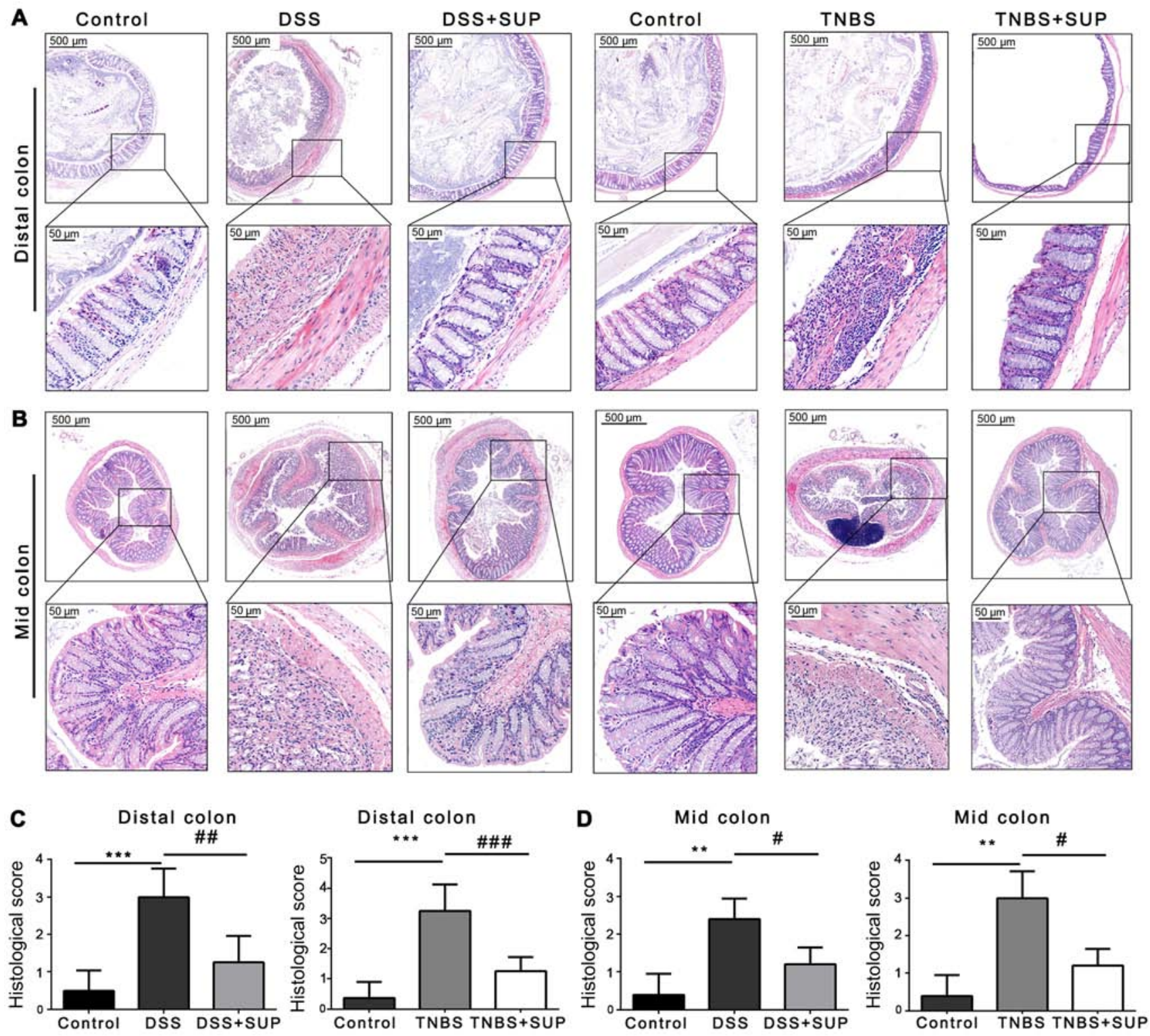

Figure 3. R. intestinalis supernatant inhibits invasion of the colon by inflammatory cells. Representative H\&E stained images of the (A) distal and (B) mid colon. Upper and lower images are at $\mathrm{x} 40$ and 200 magnification (scale bars, 500 and $50 \mu \mathrm{m}$ ). (C and D) Pathological score for the different groups ( $\mathrm{n}=6$ ). Data are representative of three independent experiments. Error bars represent mean \pm standard error of the mean. ${ }^{* *} \mathrm{P}<0.01,{ }^{* * *} \mathrm{P}<0.001 \mathrm{vs}$. control; ${ }^{\#} \mathrm{P}<0.05,{ }^{\# \#} \mathrm{P}<0.01$, ${ }^{\# \# \# P<0.001 ~ v s . ~ D S S ~ o r ~ T N B S ; ~ n s, ~ n o n-s i g n i f i c a n t ; ~ D S S, ~ d e x t r a n ~ s u l f a t e ~ s o d i u m ; ~ T N B S, ~ 2,4,6-t r i n t i r o b e n z e n e s u l f o n i c ~ a c i d ; ~ S U P, ~ s u p e r n a t a n t ~ t r e a t m e n t . ~}$

associated with the anti-inflammatory effects of $R$. intestinalis in the colitis models employed in the present study. Sodium butyrate and $R$. intestinalis supernatant had similar effects with respect to inhibiting IL-6 expression by macrophages in vitro (Fig. S2).

\section{Discussion}

Our previous study examined the anti-inflammatory properties of $R$. intestinalis bacteria; reductions in IL-17 levels were reported in an animal model of colitis treated with $R$.intestinalis and in a cellular model of inflammation (LPS-treated NCM460 colon cells co-cultured with $R$. intestinalis) (5). Additionally, $R$. intestinalis was reported to exert protective effects in a TNBS-induced colitis model by promoting Treg differentiation in the colon and the secretion of anti-inflammatory mediators, including IL-10, TSLP, and TGF- $\beta$ (4). In addition, the bacteria have similar anti-inflammatory effects on LPS-induced Caco2 colon cells (4). The findings of these aforementioned studies suggest that $R$. intestinalis could be a candidate probiotic for the treatment of IBD; however, the safety, efficacy and dose of bacteria for clinical practice remains uncertain and further investigation is required $(4,5)$. On the contrary, examining the effects of $R$. intestinalis supernatant may improve understanding of the interaction between bacteria and host, and may aid the development of safer treatment strategies for future clinical use.

The effects of $R$. intestinalis supernatant were determined in the present study. The results indicated that the supernatant inhibited the expression of IL-6 induced by LPS in RAW264.7 macrophages and BMMs in vitro. A recent study showed that the metabolites generated by the microbiota protect IECs, and serve a role in the interaction between IECs and macrophages (27). Conversely, it was reported that $R$. intestinalis 
A

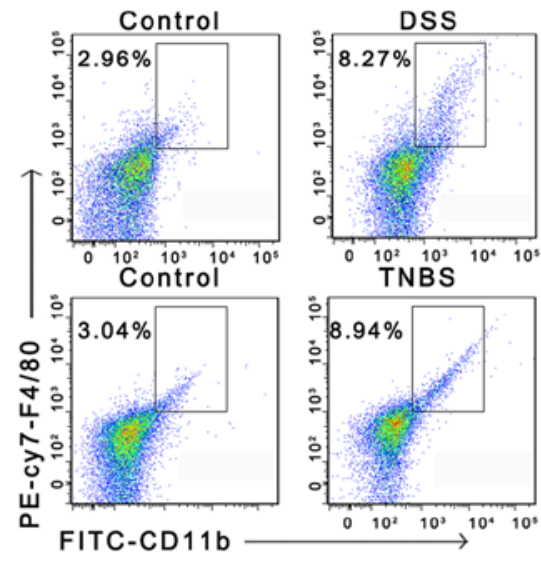

DSS+SUP
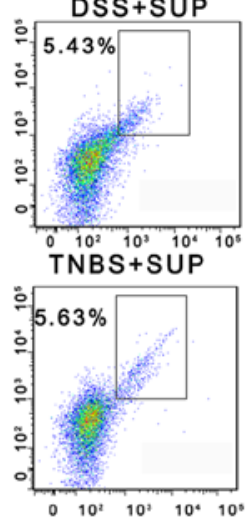

B

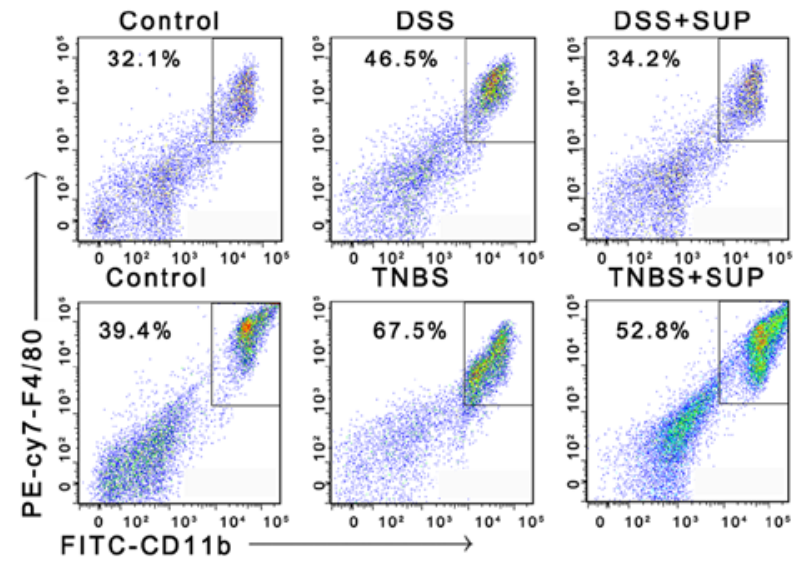

C

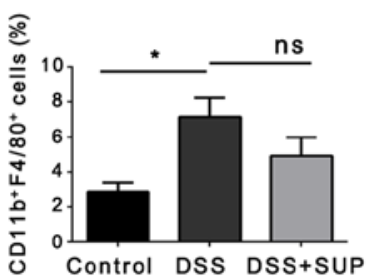

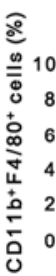

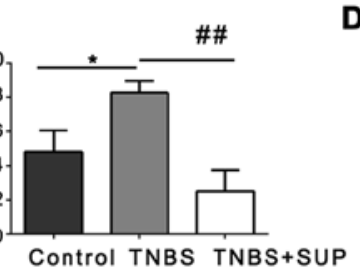

D $气$
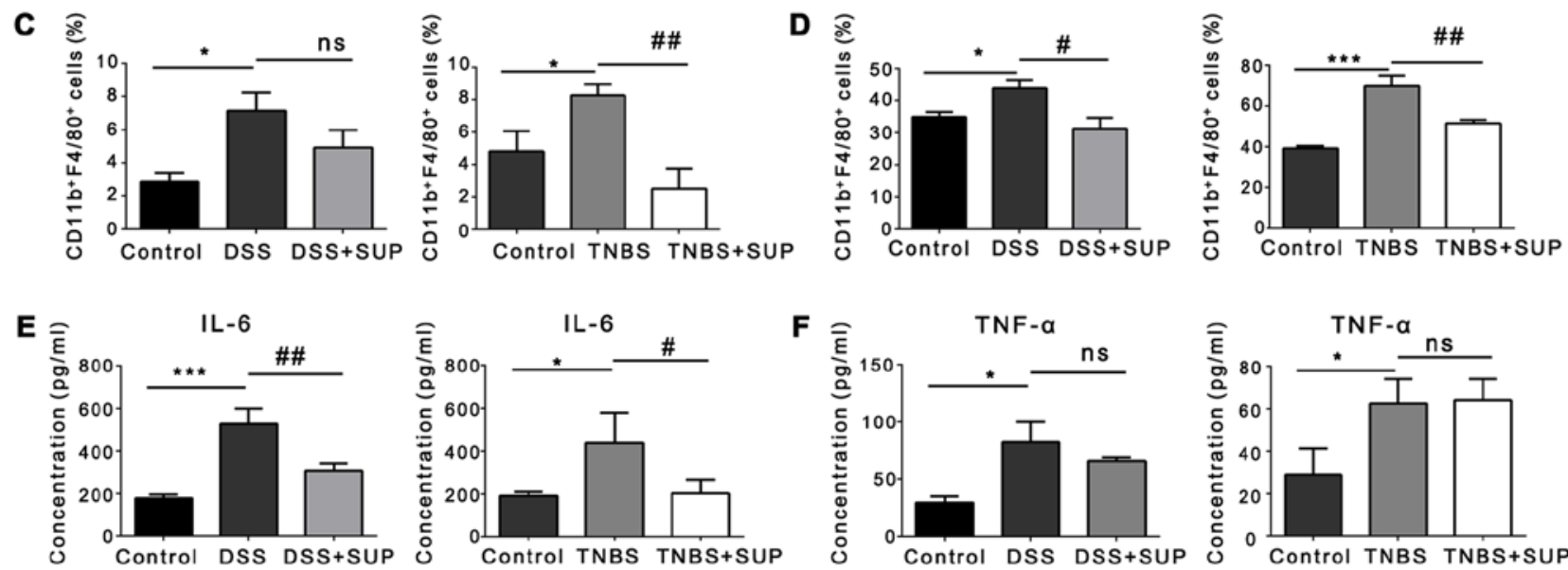

G
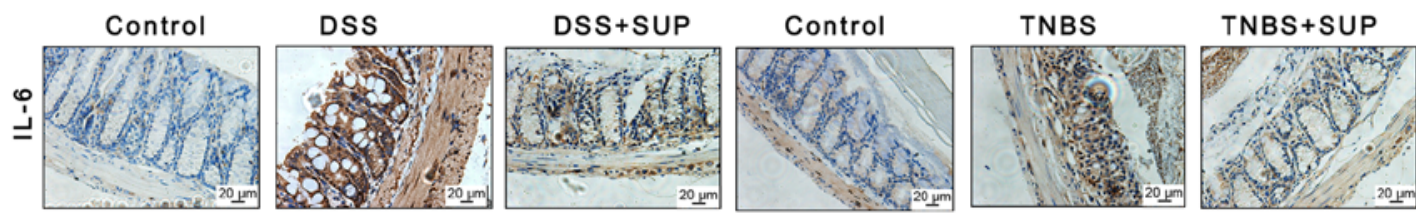

H
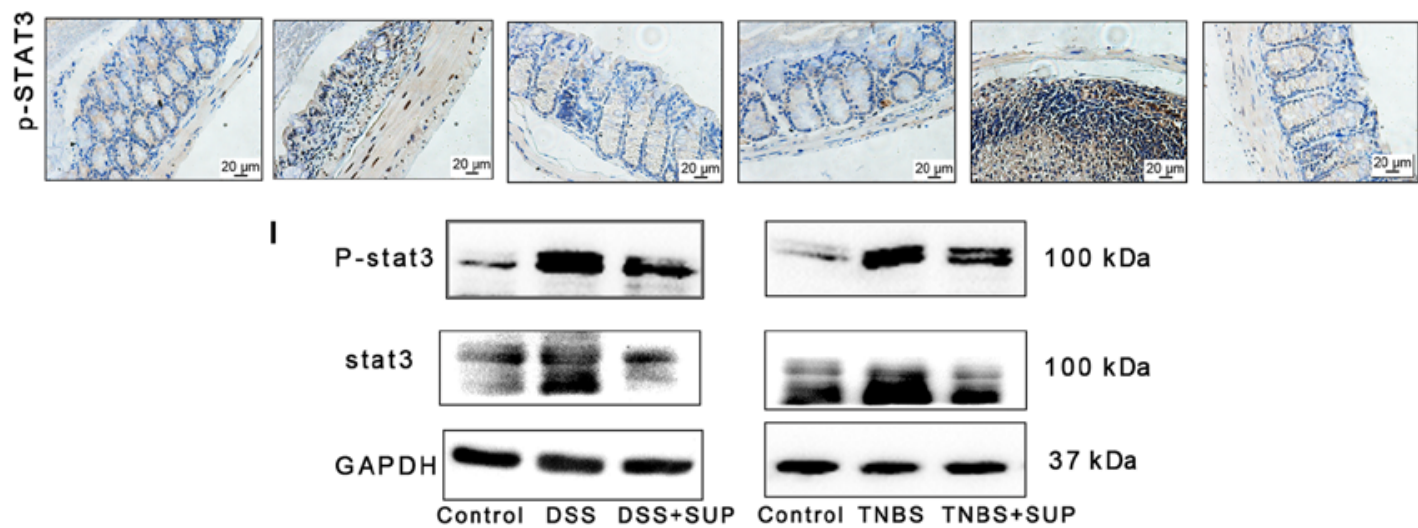

Figure 4. Fewer F4/80 ${ }^{+} \mathrm{CD} 1 \mathrm{~b}^{+}$macrophages are detected in DSS/TNBS-induced colitis mice treated with $R$. intestinalis supernatant. Representative flow cytometry plots of (A) colonic $\mathrm{F} 4 / 80^{+} \mathrm{CD} 11 \mathrm{~b}^{+}$macrophages and (B) peritoneal $\mathrm{F} 4 / 80^{+} \mathrm{CD} 11 \mathrm{~b}^{+}$macrophages $(\mathrm{n}=6)$. $(\mathrm{C}$ and $\mathrm{D})$ Statistical analysis of the number of $\mathrm{F} 4 / 80^{+} \mathrm{CD} 1 \mathrm{~b}^{+}$cells in the colon (left) and peritoneal cavity (right) $(\mathrm{n}=6$ ). ELISA of (E) IL-6 and (F) TNF- $\alpha$ levels in mouse serum ( $\mathrm{n}=6$ ). Representative immunohistochemistry images of (G) IL-6 and (H) p-STAT3 expression in colon tissues (scale bar, $20 \mu \mathrm{m}$; magnification, x200; n=6). (I) Western blot analysis of STAT3 and p-STAT3 in colon tissues. Data are representative of three independent experiments. Error bars represent mean \pm standard error of the mean. ${ }^{*} \mathrm{P}<0.05,{ }^{* * * *} \mathrm{P}<0.001$ vs. control; ${ }^{\#} \mathrm{P}<0.05,{ }^{\# \#} \mathrm{P}<0.01$ vs. DSS or TNBS; ns, non-significant; DSS, dextran sulfate sodium; IL, interleukin; $\mathrm{p}$, phosphorylated; PE, phycoerythrin; STAT3, signal transducer and activator 3; SUP, supernatant treatment; TNBS, 2,4,6-trintirobenzenesulfonic acid; TNF- $\alpha$, tumor necrosis factor- $\alpha$.

supernatant had no significant effects on IL-6 expression by $\mathrm{Caco} 2$ colon cells in vitro. Thus, it was proposed that
$R$. intestinalis supernatant exerts its function via immune cells and thus its function was investigated in vivo. 
A

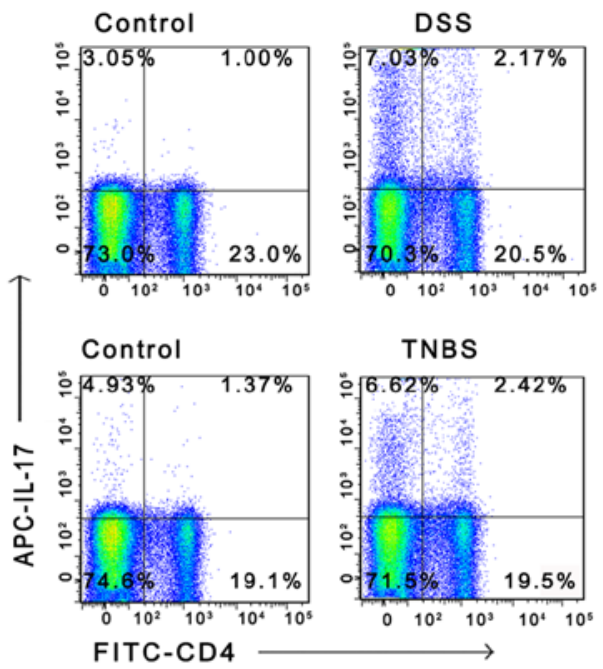

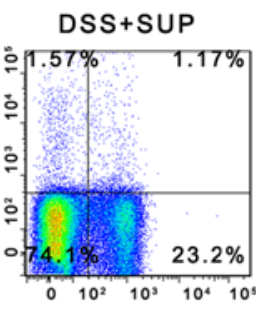

TNBS+SUP

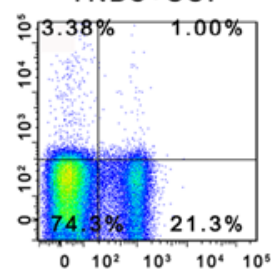

B
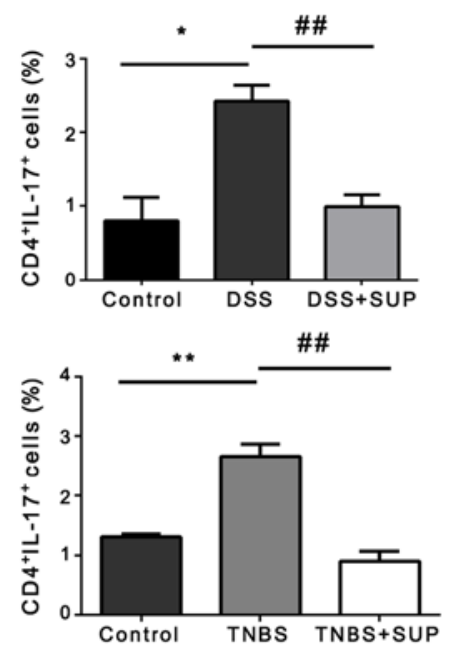

c

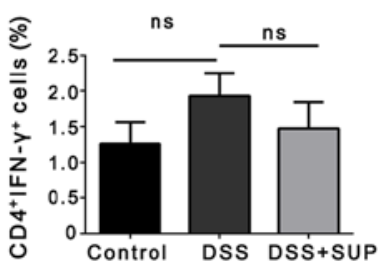

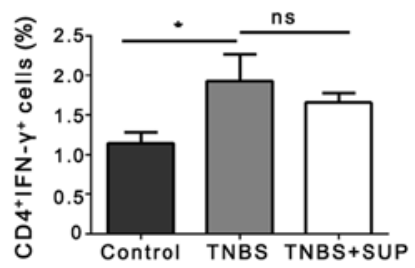
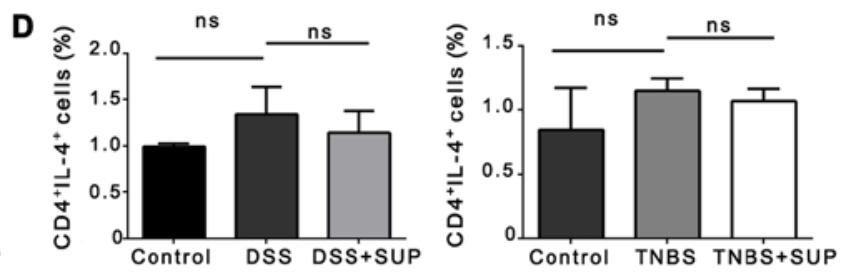

Figure 5. Fewer Th17 cells are detected in mice with colitis exposed to R. intestinalis supernatant. (A) Representative flow cytometry plots and (B) statistical analysis of the percentage of CD4+IL-17+ cells (Th17 cells) within the splenic cell population ( $\mathrm{n}=6)$. The percentage of (C) CD4 $4^{+} \mathrm{IFN}-\gamma^{+} \mathrm{T}$ cells $(\mathrm{Th} 1)$ and (D) $\mathrm{CD} 4{ }^{+} \mathrm{IL}-4^{+} \mathrm{T}$ cells $(\mathrm{Th} 2)$ in the spleen was analyzed by flow cytometry $(\mathrm{n}=6)$. Data are representative of three independent experiments. Error bars represent

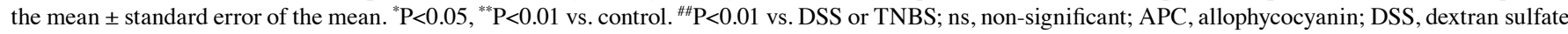
sodium; FITC, fluorescein isothiocyanate; IFN- $\gamma$, interferon- $\gamma$; IL, interleukin; SUP, supernatant treatment; TNBS, 2,4,6-trintirobenzenesulfonic acid.

In addition, the effects of the bacterial supernatant were investigated in murine models of DSS- and TNBS-induced colitis in the present study. Mice with DSS-induced colitis are considered to be a model of human UC, whereas mice with TNBS-induced colitis represent a model of human CD (18). These models are used widely to undertake research on other commensal microbiota, such as Faecalibacterium prausnitzii (11). Furthermore, compounds such as recombinant human milk fat globule epidermal growth factor VIII have been investigated in DSS- and TNBS-induced models to determine their therapeutic potential in IBD (28). The present study revealed that $R$. intestinalis supernatant ameliorated DSS- and TNBS-induced colitis. These similar findings obtained from both models suggest that $R$. intestinalis supernatant may exert anti-inflammatory effects against IBD.

Macrophages in the colonic mucosa serve vital roles in innate immune responses. The cells possess numerous functions, including phagocytosis to remove pathogenic microorganisms and apoptotic cells to maintain homeostasis, the secretion of various cytokines involved in immune regulation, the presentation of antigens to $\mathrm{T}$ cells and $\mathrm{T}$ cell activation $(29,30)$. In IBD, macrophages accumulate in the injured mucosa and secrete IL-1 $\beta$, IL- 6 and TNF- $\alpha$ to induce inflammatory responses and maintain inflammatory conditions (21). In the present study, a significant reduction in the abundance of colonic macrophages in TNBS + SUP group was observed, but not in the DSS + SUP group. These differences between the two groups may be due to the differing susceptibilities to the effects of $R$. intestinalis between the DSS- and TNBS-induced colitis models. A previous study reported that transgenic mouse expressing TNF- $\alpha$-induced protein 3 in IECs were protected from DSS-induced colitis but not TNBS-induced colitis, also indicating the different susceptibilities between DSS- and TNBS-induced colitis models (31). In addition, dynamic alterations in the number of colon macrophages in the DSS-induced colitis model may underlie these observations (32). Further investigation using flow cytometry assays of colon macrophages at several continuous time points may provide further insight. However, the present study reported an increase in the number of peritoneal inflammatory macrophages in mice with DSS- and TNBS-induced colitis. Increased IL-6 production was detected in LPS-induced macrophages in vitro but was ameliorated by $R$. intestinalis supernatant. These results suggest that $R$. intestinalis supernatant functions by regulating the activity of macrophages. Furthermore, accumulating evidence suggests that circulating or local levels of IL- 6 affect IBD, and that IL- 6 promotes the differentiation of $\mathrm{CD}^{+} \mathrm{T}$ cells into Th17 cells $(26,33)$. IL-6 is upregulated via activation of the STAT3 signaling pathway under inflammatory conditions (34). The results of the present study revealed upregulated IL-6 and STAT3 expression, and increased numbers of Th17 cells in DSS- and TNBS-induced colitis models; opposing effects were observed following treatment with $R$. intestinalis supernatant.

The components of $R$. intestinalis, such as the flagellin protein, are essential for suppressing colitis via binding to unique receptors such as Toll-like receptor 5 (TLR5) (35). To determine whether non-protein components exhibits any effects on BMMs, R. intestinalis supernatant was irradiated with UV, heated to $>100^{\circ} \mathrm{C}$ and treated with trypsin. The supernatant 


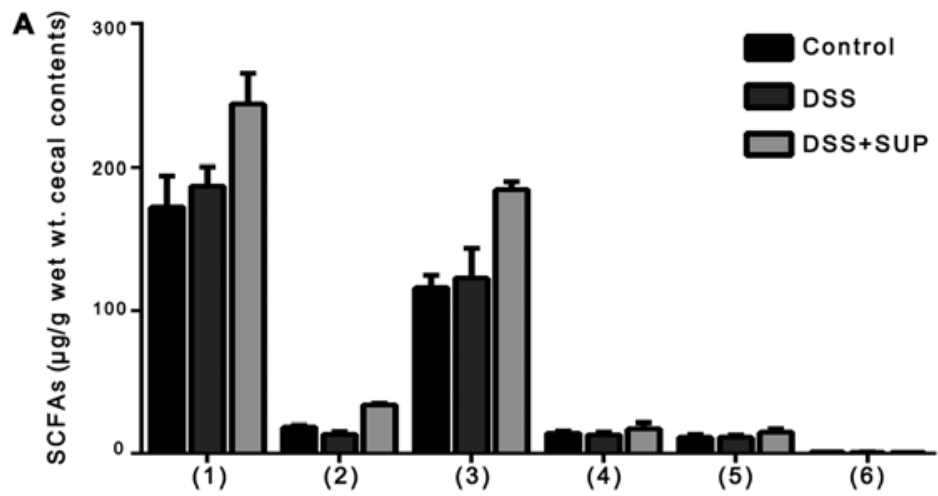

(1) Propionic acid

(2) Isobutyric acid

(3) Butyric acid

(4) Isovaleric acid

(5) Valeric acid

(6) Hexanoic acid

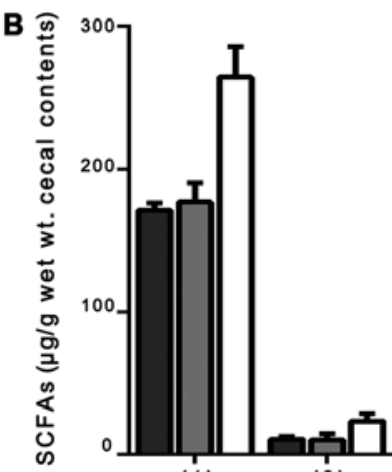

(1)
(2)

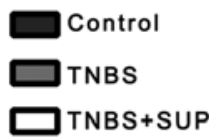

Figure 6. Gas chromatography-mass analysis of SCFAs in mouse cecal contents. (A and B) Propionic acid, isobutyric acid, butyric acid, isovaleric acid, valeric acid and hexanoic acid were detected in the cecal contents of mice $(n=6)$. Data are representative of three independent experiments. Error bars represent the mean \pm standard error of the mean. DSS, dextran sulfate sodium; SCFAs, short-chain fatty acids; TNBS, 2,4,6-trintirobenzenesulfonic acid.

was then used to treat BMMs isolated from TLR5 $5^{-/-}$mice. The results suggested that $R$. intestinalis supernatant downregulated the levels of IL-6 (Fig. S1B), supporting that $R$. intestinalis supernatant contains active non-protein components.

Additionally, GC-MS was conducted in the present study to identify substances in the $R$. intestinalis supernatant; SCFAs, including butyrate were detected. Butyrate exerts an anti-inflammatory effect on immune cells involved in inflammation; it suppresses Th9 and Th2-mediated immune responses to attenuate inflammation in the lungs (36). Furthermore, microbiota-derived butyrate inhibited the apoptosis of IECs, decreased the production of inflammatory cytokines, including interferon- $\gamma$ and IL-1 $\beta$, reduced intestinal inflammation and maintained intestinal homeostasis (37). Several studies have proposed that the molecular mechanism underlying the anti-inflammatory effects of butyrate may involve downregulating the expression of proinflammatory factors, such as NO, IL-6, and IL-12, and regulating the activity of macrophages in the lamina propria $(14,27,38)$. On the contrary, butyrate has no effect on the secretion of TNF- $\alpha$ and C-X-C motif chemokine 12 by macrophages (14). In addition, $R$. intestinalis supernatant did not significantly affect the expression of LPS-induced TNF- $\alpha$, opposing the effects of Faecalibacterium prausnitzii supernatant (39).

Finally, LPS-induced RAW264.7 macrophages were treated with the same concentration of sodium butyrate detected in the supernatant. The similar results obtained for the sodium butyrate and the $R$. intestinalis supernatant treatment groups suggest that butyrate present in $R$. intestinalis supernatant exerts anti-inflammatory effects (Fig. S2). However, further investigation using sodium butyrate as a control, and combined with a butyrate receptor inhibitor are required for further confirmation.

Collectively, the results of the present study indicated that $R$. intestinalis supernatant regulated immune responses and ameliorated DSS- and TNBS-induced colitis.

\section{Acknowledgements}

Not applicable.

\section{Funding}

The present study was supported by the National Natural Science Foundation of China (grant nos. 81670504 and 81472287) and Natural Science Foundation of Hunan (grant no. 2017JJ3468).

\section{Availability of data and materials}

The datasets used and/or analyzed during the current study are available from the corresponding author on reasonable request.

\section{Authors' contributions}

WL, BT and MX performed the experiments. CZ, LT and YQ collected the data SW, ZY, XWu and XM analyzed the data, and WL prepared the manuscript. ZS, MD and $\mathrm{XL}$ revised the manuscript and contributed to study design. XWa designed the study and supervised the experiments. All authors read and approved the final manuscript. 


\section{Ethics approval and consent to participate}

All animal experiments were approved by the Ethical Committee of Medical Research of Central South University.

\section{Patient consent for publication}

Not applicable.

\section{Competing interests}

The authors declare that they have no competing interests.

\section{References}

1. Torres J, Mehandru S, Colombel JF and Peyrin-Biroulet L: Crohn's disease. Lancet 389: 1741-1755, 2017.

2. Ungaro R, Mehandru S, Allen PB, Peyrin-Biroulet L and Colombel JF: Ulcerative colitis. Lancet 389: 1756-1770, 2017.

3. de Souza HSP, Fiocchi C and Iliopoulos D: The IBD interactome: An integrated view of aetiology, pathogenesis and therapy. Nat Rev Gastroenterol Hepatol 14: 739-749, 2017.

4. Shen Z, Zhu C, Quan Y, Yang J, Yuan W, Yang Z, Wu S, Luo W, Tan B and Wang X: Insights into Roseburia intestinalis which alleviates experimental colitis pathology by inducing anti-inflammatory responses. J Gastroenterol Hepatol 33: 1751-1760, 2018.

5. Zhu C, Song K, Shen Z, Quan Y, Tan B, Luo W, Wu S, Tang K, Yang Z and Wang X: Roseburia intestinalis inhibits Interleukin17 Excretion and promotes regulatory $\mathrm{T}$ cells differentiation in colitis. Mol Med Rep 17: 7567-7574, 2018.

6. Duncan SH, Hold GL, Barcenilla A, Stewart CS and Flint HJ: Roseburia intestinalis sp. nov., a novel saccharolytic butyrate-producing bacterium from human faeces. Int J Syst Evol Microbiol 52: 1615-1620, 2002.

7. Cani PD: Human gut microbiome: Hopes, threats and promises. Gut 67: 1716-1725, 2018.

8. Goncalves P, Araujo JR and Di Santo JP: A Cross-talk between Microbiota-derived short-chain fatty acids and the host mucosal immune system regulates intestinal homeostasis and inflammatory bowel disease. Inflamm Bowel Dis 24: 558-572, 2018.

9. Arpaia N, Campbell C, Fan X, Dikiy S, van der Veeken J, deRoos P, Liu H, Cross JR, Pfeffer K, Coffer PJ and Rudensky AY: Metabolites produced by commensal bacteria promote peripheral regulatory T-cell generation. Nature 504: 451-455, 2013.

10. Singh N, Gurav A, Sivaprakasam S, Brady E, Padia R, Shi H, Thangaraju M, Prasad PD, Manicassamy S, Munn DH, et al: Activation of Gpr109a, receptor for niacin and the commensal metabolite butyrate, suppresses colonic inflammation and carcinogenesis. Immunity 40: 128-139, 2014

11. Zhou L, Zhang M, Wang Y, Dorfman RG, Liu H, Yu T, Chen X, Tang D, Xu L, Yin Y, et al: Faecalibacterium prausnitzii produces butyrate to maintain Th17/Treg balance and to ameliorate colorectal colitis by inhibiting histone deacetylase 1. Inflamm Bowel Dis: May 23, 2018 (Epub ahead of print). doi: 10.1093/ibd/izy182.

12. National Research Council (US) Committee for the Update of the Guide for the Care and Use of Laboratory A: The National Academies Collection: Reports funded by National Institutes of Health. In: Guide for the Care and Use of Laboratory Animals. 8th edition. National Academies Press (US), Washington, DC, 2011.

13. Murray PJ, Allen JE, Biswas SK, Fisher EA, Gilroy DW, Goerdt S, Gordon S, Hamilton JA, Ivashkiv LB, Lawrence T, et al: Macrophage activation and polarization: Nomenclature and experimental guidelines. Immunity 41: 14-20, 2014.

14. Chang PV, Hao L, Offermanns S and Medzhitov R: The microbial metabolite butyrate regulates intestinal macrophage function via histone deacetylase inhibition. Proc Natl Acad Sci USA 111: 2247-2252, 2014.

15. Livak KJ and Schmittgen TD: Analysis of relative gene expression data using real-time quantitative PCR and the 2(-Delta Delta C(T)) method. Methods 25: 402-408, 2001.

16. Turnbaugh PJ, Ley RE, Mahowald MA, Magrini V, Mardis ER and Gordon JI: An obesity-associated gut microbiome with increased capacity for energy harvest. Nature 444: 1027-1031, 2006.
17. Samuel BS and Gordon JI: A humanized gnotobiotic mouse model of host-archaeal-bacterial mutualism. Proc Natl Acad Sci USA 103: 10011-10016, 2006.

18. Wirtz S, Popp V, Kindermann M, Gerlach K, Weigmann B, Fichtner-Feigl $S$ and Neurath MF: Chemically induced mouse models of acute and chronic intestinal inflammation. Nat Protoc 12: 1295-1309, 2017.

19. Dale DC, Boxer L and Liles WC: The phagocytes: Neutrophils and monocytes. Blood 112: 935-945, 2008.

20. Sica A and Mantovani A: Macrophage plasticity and polarization: In vivo veritas. J Clin Invest 122: 787-795, 2012.

21. Gren ST and Grip O: Role of monocytes and intestinal macrophages in crohn's disease and ulcerative colitis. Inflamm Bowel Dis 22: 1992-1998, 2016.

22. Zhou B, Xia X, Wang P, Chen S, Yu C, Huang R, Zhang R, Wang Y, Lu L, Yuan F, et al: Induction and amelioration of methotrexate-induced gastrointestinal toxicity are related to immune response and gut microbiota. EBioMedicine 33: 122-133, 2018.

23. Mitsuyama K, Matsumoto S, Masuda J, Yamasakii H, Kuwaki K, Takedatsu $\mathrm{H}$ and Sata M: Therapeutic strategies for targeting the IL-6/STAT3 cytokine signaling pathway in inflammatory bowel disease. Anticancer Res 27: 3749-3756, 2007.

24. Imam T, Park S, Kaplan MH and Olson MR: Effector T helper cell subsets in inflammatory bowel diseases. Front Immunol 9: 1212, 2018.

25. Chen ML and Sundrud MS: Cytokine networks and T-Cell subsets in inflammatory bowel diseases. Inflamm Bowel Dis 22: 1157-1167, 2016.

26. Nagashima $\mathrm{H}$, Ishii $\mathrm{N}$ and So T: Regulation of interleukin- 6 receptor signaling by TNF receptor-associated factor 2 and 5 during differentiation of inflammatory CD4(+) T cells. Front Immunol 9: 1986, 2018.

27. Chen G, Ran X, Li B, Li Y, He D, Huang B, Fu S, Liu J and Wang W: Sodium butyrate inhibits inflammation and maintains epithelium barrier integrity in a TNBS-induced inflammatory bowel disease mice model. EBioMedicine 30: 317-325, 2018.

28. Zhang Y, Brenner M, Yang WL and Wang P: Recombinant human MFG-E8 ameliorates colon damage in DSS- and TNBS-induced colitis in mice. Lab Invest 95: 480-490, 2015.

29. Gordon S: Alternative activation of macrophages. Nat Rev Immunol 3: 23-35, 2003.

30. Mosser DM and Edwards JP: Exploring the full spectrum of macrophage activation. Nat Rev Immunol 8: 958-969, 2008.

31. Rhee L, Murphy SF, Kolodziej LE, Grimm WA, Weber CR, Lodolce JP, Chang JE, Bartulis SJ, Messer JS, Schneider JR, et al: Expression of TNFAIP3 in intestinal epithelial cells protects from DSS-but not TNBS-induced colitis. Am J Physiol Gastrointest Liver Physiol 303: G220-G227, 2012.

32. Wang W, XY L, Zheng D, Zhang D, Huang S, Zhang X, Ai F, Wang X, Ma J, Xiong W, et al: Dynamic changes of peritoneal macrophages and subpopulations during ulcerative colitis to metastasis of colorectal carcinoma in a mouse model. Inflamm Res 62. 669-680, 2013.

33. Bettelli E, Carrier Y, Gao W, Korn T, Strom TB, Oukka M, Weiner HL and Kuchroo VK: Reciprocal developmental pathways for the generation of pathogenic effector TH17 and regulatory T cells. Nature 441: 235-238, 2006.

34. Hodge DR, Hurt EM and Farrar WL: The role of IL-6 and STAT3 in inflammation and cancer. Eur J Cancer 41: 2502-2512, 2005.

35. Quan Y, Song K, Zhang Y, Zhu C, Shen Z, Wu S, Luo W, Tan B, Yang $\mathrm{Z}$ and Wang X: Roseburia intestinalis-derived flagellin is a negative regulator of intestinal inflammation. Biochem Biophys Res Commun 501: 791-799, 2018

36. Vieira RS, Castoldi A, Basso PJ, Hiyane MI, Câmara NOS and Almeida RR: Butyrate attenuates lung inflammation by negatively modulating Th9 cells. Front Immunol 10: 67, 2019.

37. Zhong X, Zhang Z, Wang S, Cao L, Zhou L, Sun A, Zhong Z and Nabben M: Microbial-Driven butyrate regulates Jejunal homeostasis in piglets during the weaning stage. Front Microbiol 9: 3335, 2019.

38. Chakravortty D, Koide N, Kato Y, Sugiyama T, Mu MM, Yoshida T and Yokochi T: The inhibitory action of butyrate on lipopolysaccharide-induced nitric oxide production in RAW 264.7 murine macrophage cells. J Endotoxin Res 6: 243-247, 2000.

39. Martin R, Chain F, Miquel S, Lu J, Gratadoux JJ, Sokol H, Verdu EF, Bercik P, Bermudez-Humaran LG and Langella P: The commensal bacterium Faecalibacterium prausnitzii is protective in DNBS-induced chronic moderate and severe colitis models. Inflamm Bowel Dis 20: 417-430, 2014.

This work is licensed under a Creative Commons Attribution-NonCommercial-NoDerivatives 4.0 International (CC BY-NC-ND 4.0) License. 